\title{
Entre ombre et lumière, analyse communicationnelle de la construction identitaire de l'ONG Finance Watch
}

Ce que l'analyse des écrits dit sur l'énonciation de l'organisation Between light and shade: an analysis in communication of the identity construction of the NGO Finance Watch. What the analysis of written texts says of the organization statement

\section{Sandrine Roginsky et François Lambotte}

\section{(2) OpenEdition}

\section{Journals}

Édition électronique

URL : http://journals.openedition.org/communicationorganisation/4572

DOI : 10.4000/communicationorganisation.4572

ISSN : $1775-3546$

\section{Éditeur}

Presses universitaires de Bordeaux

\section{Édition imprimée}

Date de publication : 1 juin 2014

Pagination : 175-192

ISBN : 978-2-86781-904-9

ISSN : $1168-5549$

\section{Référence électronique}

Sandrine Roginsky et François Lambotte, « Entre ombre et lumière, analyse communicationnelle de la construction identitaire de l'ONG Finance Watch », Communication et organisation [En ligne], 45 | 2014, mis en ligne le 01 juin 2017, consulté le 30 avril 2019. URL : http://journals.openedition.org/ communicationorganisation/4572 ; DOI : 10.4000/communicationorganisation.4572 


\title{
Entre ombre et lumière, analyse communicationnelle de la construction identitaire de l'ONG Finance Watch \\ Ce que l'analyse des écrits dit sur l'énonciation de l'organisation
}

\author{
Sondrine Roginskụ' et fronçois Lambotte ${ }^{2}$
}

\section{Introduction}

Nous souhaitons dans cet article aborder la question de la transparence dans le processus de création d'une organisation. Plus particulièrement, nous nous intéressons aux premiers pas de l'ONG "Finance Watch ", le seul contrelobby dans le secteur bancaire. Finance Watch (FW) est une association créée à Bruxelles en 2011 pour permettre à la société civile européenne de produire une expertise sur les questions financières qui puisse rivaliser avec celle des différents groupes d'intérêt travaillant pour l'industrie financière.

Pour comprendre cet enjeu, nous proposons d'analyser ces premiers moments en nous penchant sur la fabrique d'écrits stables. La conception de ces premiers discours (dossier de presse, statuts, présentation, etc.) a fait l'objet de choix multiples construisant progressivement une certaine "image " de l'organisation. En nous interrogeant sur les pratiques d'écriture et ce qu'elles peuvent produire à la genèse d'une organisation, nous souhaitons d'abord comprendre l'incidence du contexte, des conditions de production sur la construction identitaire de l'organisation. L'écriture est socialement située dans un contexte spécifique, ici celui du Parlement européen et de la société civile européenne. En ce sens, le contexte contraint l'écriture et les acteurs dans leur négociation de l'écrit (Delcambre, 1990a ; Pène 1995). En parallèle, nous nous interrogeons sur les stratégies de communication et d'expression des acteurs de l'écriture (Delcambre, 1990a) car « étudier les écrits, c'est

1 Sandrine Roginsky est docteure en sociologie, professeure à l'école de communication de l'Université Catholique de Louvain (Belgique). Elle est chercheure au laboratoire d'analyse des systèmes de communication des organisations (LASCO); sandrine.roginsky@uclouvain.be

2 François Lambotte est docteur en sciences de gestion, professeur à l'école de communication de l'Université Catholique de Louvain (Belgique). Il est président du laboratoire d'analyse des systèmes de communication des organisations (LASCO); francois.lambotte@uclouvain.be 


\section{$\mathrm{C} \& O \mathrm{n}^{\circ} 45$}

prendre en compte la simultanéité des interactions humaines, c'est suivre la successivité du procès » (Pène 1995 p. 6).

Cette analyse permet, selon nous, d'identifier ce qui à travers la fabrique de différents écrits stables à la genèse de la création FW a fait l'objet de choix, volontaire ou non, de mise en lumière ou de mise à l'écart d'éléments constitutifs de l'organisation par les participants de la conception à ces écrits.

\section{La mise en visibilité analysée comme un processus de sélection d'information, d'énonciation et de compréhension}

Notre recherche s'inscrit dans l'approche CCO considérant la communication comme constitutive de l'organisation (Ashcraft, Kuhn \& Cooren 2009). Plus précisément, la méthode expérimentale proposée se base sur la conception systémique de la communication de Luhmann (1992) et se place dans la même veine que les travaux portés notamment par Seidl (2005a, 2005b) et Shoeneborn (2011). Afin d'expliquer la méthode, nous passons en revue les postulats que la conception de Luhmann impose. Pour chacun d'entre eux, nous développons plus précisément ce qu'ils impliquent conceptuellement et méthodologiquement.

1. Pour Luhmann (1992 p. 301), « seule la communication peut communiquer et c'est seulement dans le cadre de ce réseau de communications que ce que l'on comprend comme action est créée ». Ainsi c'est en analysant les communications à la genèse de l'organisation que nous pourrons saisir l'action de constitution de l'organisation.

2. Tout comme la vie ou la conscience (p. 302), « la communication est une réalité émergente, un état des lieux sui generis. Elle surgit de la synthèse entre trois différentes sélections, nommément, une sélection d'information, une sélection d'énonciation de cette information et une sélection de compréhension ou d'incompréhension de l'énonciation de cette information. "Ainsi c'est à travers cette sélection d'information et d'énonciation et la compréhension qui en découle que nous analyserons la mise en lumière ou l'occultation lors de la communication sur la création de cette organisation.

Pour cette étude, nous analysons les trois écrits produits à la genèse de FW. Ces trois écrits ont été sélectionnés pour leur rôle central et décisif dans l'élaboration de l'organisation. Le premier est l'appel des députés européens qui est à l'origine de la création de l'organisation. En juin 2010, 22 députés européens lancent ainsi cet appel à la société civile pour qu'elle s'organise pour créer un contre-pouvoir au lobby unidimensionnel des banques. Les statuts de l'organisation constituent le deuxième écrit étudié comme véritable socle sur lequel l'ONG se construit. Le troisième écrit inclus est le dossier de presse qui vise à présenter l'organisation à sa naissance. Il s'agit du dernier document précédant l'assemblée générale constituante de l'organisation.

Dans un premier temps, le travail d'analyse se fait verticalement au niveau de chaque écrit produit. Nous nous intéressons ici aux différents cycles texte- 
conversation qui mènent à la production de différentes versions des trois écrits stables sélectionnés. C'est le cycle à travers lequel des conversations se traduisent en un texte et inversément comment un texte se traduit dans des actes de conversations (Taylor et al., 1996). À travers l'analyse des versions et des conversations portant sur chacune d'entre elles, l'enjeu est d'identifier les moments de sélection d'abord d'information et d'énonciation dans le système de communication afin de mettre en évidence la sélection de compréhension ou d'incompréhension de ce qu'est FW au moment de son lancement.

3. Luhmann considère la communication comme un système qui a pour caractéristique de s'autogénérer. Autrement dit, et pour reprendre un terme qu'il emprunte à la biologie, la communication est une autopoï̀se, en interdépendance avec son environnement mais donc aussi très autonome. En ce sens, nous faisons l'hypothèse que les écrits stables analysés et les conversations qui les lient les uns aux autres forment un système. La fabrique de chaque écrit à travers un ensemble d'interactions forme un microsystème. Ensemble, ces systèmes interconnectés forment un mesosystème que nous considérons comme constitutif de l'organisation (Shoeneborn, 2011). Il convient dès lors d'analyser horizontalement les trois processus de sélection. En effet, c'est seulement en comparant la production du deuxième écrit avec la première (et ainsi de suite) que l'on verra s'exprimer une certaine forme de compréhension ou d'incompréhension de la première sélection d'énonciation d'information à laquelle il sera fait référence.

4. Pour Luhmann (p. 304-305), « la communication mène à une décision à savoir si l'information énoncée et comprise est acceptée ou rejetée. Un message est cru ou non... La communication force à une prise de décision qui n'aurait pas eu lieu sans communication. En ce sens, toute communication implique une certaine prise de risque. » Lors du lancement d'une organisation comme Finance Watch, la prise de risque est grande. L'enjeu est la poursuite de la communication (la fonction par essence d'un lobby étant d'exprimer un intérêt et ainsi d'agir sur la prise de décision publique). Dès lors la continuité de l'ONG dépend de sa capacité à produire et reproduire ce système de communication. La prise de risque et la volonté de faire perdurer ce système forcent la sélection et donc le choix de mettre en lumière ou d'occulter des éléments.

5. Elle permet de mettre en évidence ce que Shoeneborn (2011) nomme l'oscillation entre la visibilité et l'invisibilité des alternatives à une décision prise. Nous pensons notamment pouvoir montrer les identités possibles de FW, la sélection opérée ou non entre ces identités ou leurs composants, la sélection opérée ou non dans la mise en visibilité de ces alternatives dans le discours produit. Ce travail de communication sur son identité, ce qu'elle est, implique de communiquer sur ce qu'elle n'est pas. Mais cela n'exclut pas l'occultation potentielle de certains éléments qui impliquerait par exemple son 
rejet. La réduction du risque de rejet inciterait donc l'organisation à occulter certaines informations ou à les énoncer de façon plus ambiguë.

6. Notons que l'analyse de la communication telle que définie ici ne tient pas compte des motifs ou des intentions des acteurs de la décision de sélection. En effet, ceux-ci n'ont aucun effet sur l'organisation, seules les décisions - les communications - ont un effet sur la constitution de l'organisation (Seidl 2005b). Seul ce qui est communiqué compte. Cela évite dans un premier temps, toute forme de biais interprétatif mais cela limite également la portée de l'analyse si les traces ne sont pas conservées. En l'occurrence dans le cadre de ce travail, nous avons eu accès à des documents qui, pour leur version intermédiaire, ne sont pas publics ; l'un de nous ayant participé à l'élaboration de l'organisation.

\section{Analyse des documents : synthèse des résultats}

Nous présentons ici les principaux résultats obtenus à partir de l'analyse du cycle d'écriture des trois documents fondateurs de l'organisation. Comme le soulignent Huët et Loneux (2008), « les écrits formalisent et accompagnent les processus d'organisation sociale, ils sont le lieu où se construisent les acteurs et l'organisation elle-même ».

\section{L'appel : positionnement des auteurs et identification des acteurs en présence}

Le projet d'organisation a vu le jour dans les enceintes du Parlement européen, sous la forme d'un texte élaboré et porté d'abord par un seul député européen puis par 22 députés européens qui le rejoignent. Ce texte est l'objet d'un travail de négociation et de coordination qui se fait le plus souvent en coulisses, via des conversations orales. Le processus d'écriture du texte suit ainsi le chemin suivant :

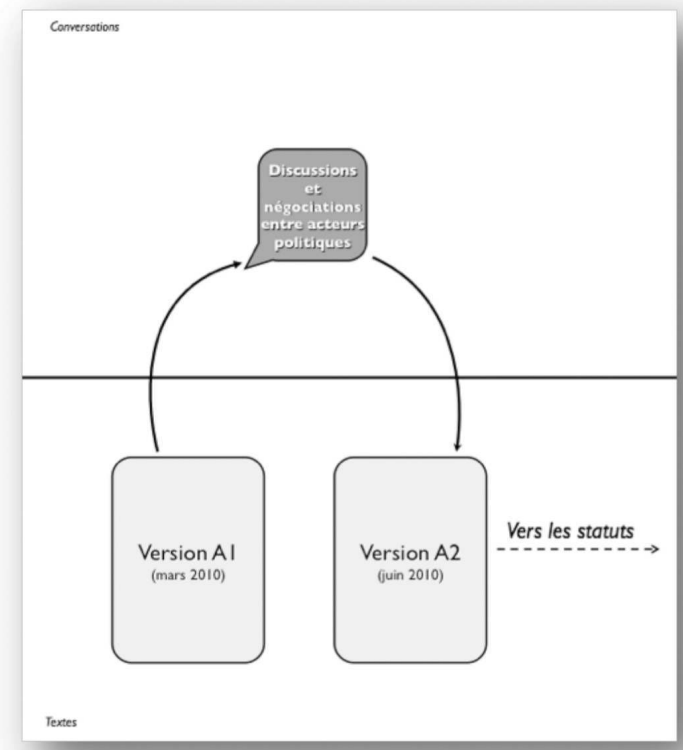

Figure 1 : Cycle texteconversation reconstitué pour l'écriture de l'appel (Sandrine Roginsky, François Lambotte) 
Appliqué à l'analyse des documents écrits qui ont abouti à l'appel pour Finance Watch, l'étude systématique du processus de sélection d'information, d'énonciation et de compréhension permet d'identifier les changements majeurs entre les textes A1 et A2 (voir tableau 1) - A pour Appel. Une première différence porte sur le soutien nécessaire à cet appel. Dans la version A1, il se mesure de manière qualitative par une adhésion des particuliers et quantitative par un seuil qui sert ainsi à évaluer le succès de la mission. Dans cette version, l'appel a donc vocation à être signé par des particuliers et des élus. Dans la version A2, seuls les élus sont ciblés par l'appel et plus aucun seuil n'est précisé. Par la sélection d'information (la suppression de l'appel à signatures), les auteurs font le choix de ne pas conditionner la création de l'organisation à un soutien citoyen. On voit ainsi pointer la disparition du citoyen, qui se confirmera par après.

Une autre différence significative concerne le positionnement des élus européens. Dans A1, ils semblent partie prenante dans le projet : " nous sommes prêts à contribuer à la constitution d'une telle ONG "; " nous travaillerons alors avec l'ensemble des organisations de la société civile». Dans A2, ils se limitent à appeler la société à s'organiser (elle-même) pour créer une telle organisation. Ils prennent ainsi leur distance. Dans la sélection de l'énonciation, on voit s'amorcer l'effacement progressif du politique dans le processus au profit des organisations de la société civile.

\begin{tabular}{|l|l|l|l|}
\hline Versions & $\begin{array}{l}\text { Sélection } \\
\text { d'information }\end{array}$ & $\begin{array}{l}\text { Sélection } \\
\text { d'énonciation }\end{array}$ & $\begin{array}{l}\text { Sélection de } \\
\text { compréhension }\end{array}$ \\
\hline $\mathrm{A} 1 \rightarrow \mathrm{A} 2$ & & $\mathrm{X}$ & Sans incidence \\
\hline $\mathrm{A} 1 \rightarrow \mathrm{A} 2$ & $\mathrm{X}$ & & $\begin{array}{l}\text { Renforcer } \\
\text { l'expression de la } \\
\text { volonté politique }\end{array}$ \\
\hline $\mathrm{A} 1 \rightarrow \mathrm{A} 2$ & $\mathrm{X}$ & & $\begin{array}{l}\text { Disparition du } \\
\text { soutien citoyen }\end{array}$ \\
\hline $\mathrm{A} 1 \rightarrow \mathrm{A} 2$ & & $\mathrm{X}$ & $\begin{array}{l}\text { Effacement du } \\
\text { politique }\end{array}$ \\
\hline
\end{tabular}

Tableau 1 - Processus de sélection d'information, d'énonciation et de compréhension appliqué à l'écriture de "l'appel pour Finance Watch » (Sandrine Roginsky, François Lambotte)

\section{Les statuts : lissage du discours, disparition des acteurs / auteurs et rhétorique de l'expertise}

Nous pouvons identifier neuf documents qui constituent les différentes étapes de l'écriture des statuts : les deux premières versions des statuts ont comme auteurs des acteurs politiques; les sept autres versions émanent de «Finance Watch » mais n'ont pas d'auteurs identifiés. Un chargé de projet 


\section{$\mathrm{C} \& O \mathrm{n}^{\circ} 45$}

Finance Watch a en effet émergé entre les versions S2 et S3 - S pour Statut, mais son nom n'apparaît jamais sur les documents étudiés. Il ne faut pas voir l'écriture des documents statutaires de manière isolée, mais plutôt les remettre dans un contexte plus global de conversations et d'écritures qui précèdent la conception de ce document. Autrement dit, le texte de l'appel présenté plus haut précède et inspire l'écriture des statuts. C'est la raison pour laquelle il est nécessaire d'étudier ces textes dans la continuation des textes passés (axe horizontal de l'analyse). L'évolution des documents écrits suit le calendrier ci-dessous, ponctué par des évènements de nature orale :

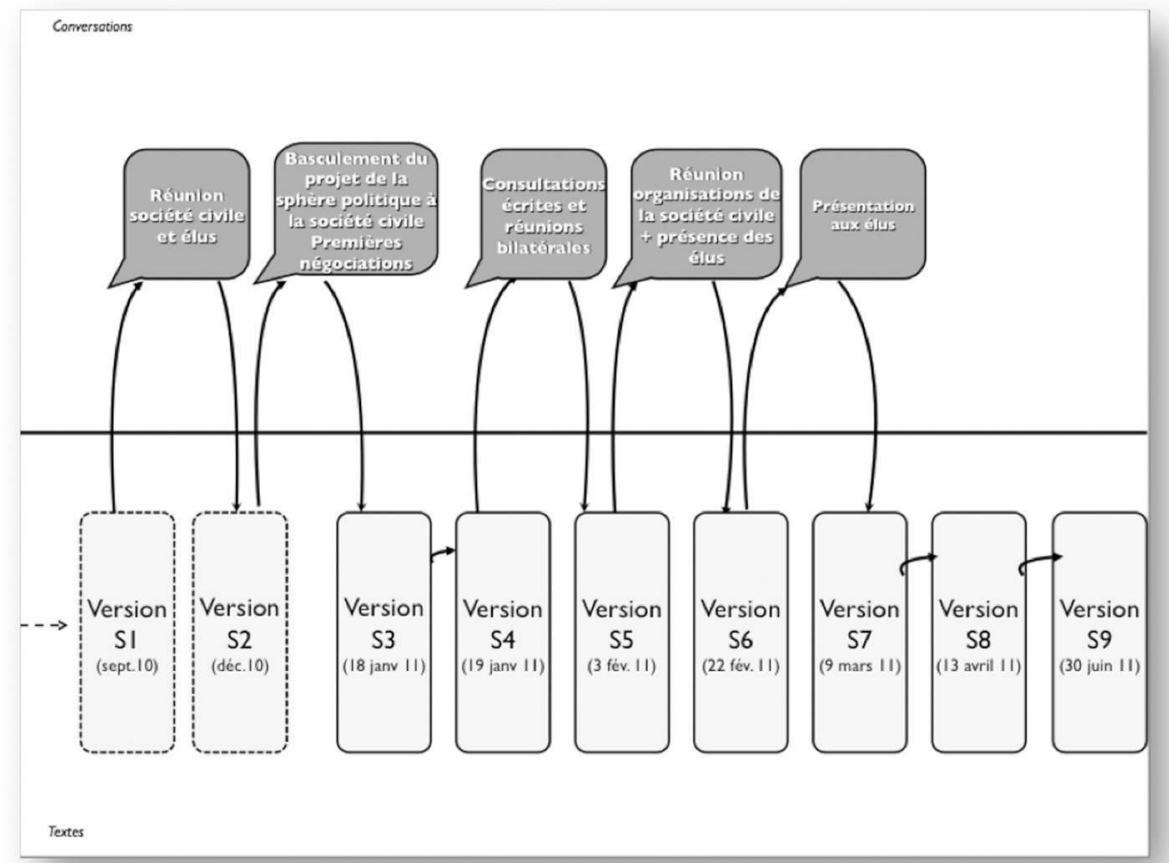

Figure 2 : Cycle texte-conversation reconstitué pour l'écriture des statuts (Sandrine Roginsky, François Lambotte)

L'analyse comparée des documents permet de souligner plusieurs caractéristiques majeures :

- La disparition de la «société civile»

Le premier document n'a pas d'auteur désigné mais émane du bureau du député initiateur. Il sert de base aux premières discussions avec les organisations de la société civile. Il rappelle l'initiative de l'appel et les deux objectifs prioritaires d'une future organisation Finance Watch : le lobbying et l'expertise. Cependant des nuances sont apportées par rapport au texte de l'appel. En effet dans le texte de l'appel il est mentionné une " absence de contre-expertise » de la société civile face au lobbying de l'industrie 
financière. Or dans le texte qui pose les premières bases de ce qui deviendra ensuite le document présentant les statuts de l'organisation, cette remarque est nuancée puisqu'il est cette fois précisé que les ONG de la société civile produisent de l'expertise, d'une quantité décente qui plus est. À ce stade pourtant, les politiques n'ont pas encore rencontré les organisations de la société civile mais d'ores et déjà ils tempèrent le constat présenté dans l'appel. Ce changement d'énonciation semble participer à légitimer le basculement entre élus d'un côté et société civile de l'autre : puisque les organisations de la société civile sont d'ores et déjà capables de produire de l'expertise sur les aspects financiers, elles devraient être d'autant plus en capacité de mettre en place une organisation qui se focaliserait sur la régulation financière au niveau européen. Pourtant, au fil du processus d'écriture, elles disparaissent. En effet, le concept même de "société civile » devient de moins en moins visible. Le terme «société civile " n'apparaît finalement plus que deux fois dans la version S5 pour disparaître dans la version S6. Entre ces deux versions a lieu une réunion avec tous les acteurs concernés (autrement dit les organisations de ladite société civile) pour discuter des projets de statuts.

Finalement, à l'issue du processus d'écriture (voir tableau 2 page suivante), plus aucun élément dans les statuts ne permet de spécifier que l'organisation se donne comme mandat d'agir au nom de la société civile, sans plus d'éléments sur le type d'acteurs qui constituent Finance Watch. La sélection d'informations entraîne la disparition du concept de "société civile ", tandis que la sélection de l'énonciation fait le choix d'une terminologie plus évasive : les auteurs décident de ne plus se définir de façon précise au regard de la société civile. De même, ceux-ci ne font plus référence à l'action de campagne au nom de la société civile.

- La disparition du «public »

Nous avons constaté un peu plus tôt la disparition des citoyens dans l'écriture de l'appel : ils sont d'abord la cible de l'appel avant de n'être plus directement concernés par le texte. Un parallèle peut être dressé avec l'écriture des statuts. En effet, les premières versions prévoient un statut spécifique pour les membres individuels, autrement dit les citoyens. Mais l'analyse des documents permet de mettre en lumière la disparition des membres individuels (les citoyens). Cette décision est justifiée par un document (avec auteur cette fois) qui donne les résultats de la consultation avec les acteurs concernés par la création de l'organisation : ceux-ci représentant pour la grande majorité des organisations non gouvernementales (ONG) et associations. Ils soulignent qu'une organisation ne peut pas peser le même poids qu'un particulier. Cependant, à ce stade, un autre statut est prévu pour les individus (non spécialistes, issus du grand public) qui ne confère pas cette fois un statut de membre mais malgré tout une forme de reconnaissance, celui d'« ami de Finance Watch ». Au fur à mesure de l'évolution des documents, le statut de non membre « ami de Finance Watch » se précise en limitant fortement 


\section{$C \& O n^{\circ} 45$}

son champ d'action et en l'orientant davantage vers le rôle de donateur. Il convient par ailleurs d'ajouter ici que ces précisions ne figurent finalement pas dans les statuts définitifs et officiels.

\begin{tabular}{|l|l|l|l|}
\hline Versions & $\begin{array}{l}\text { Sélection } \\
\text { d'information }\end{array}$ & $\begin{array}{l}\text { Sélection } \\
\text { d'énonciation }\end{array}$ & $\begin{array}{l}\text { Sélection de } \\
\text { compréhension }\end{array}$ \\
\hline $\mathrm{A} 2 \rightarrow \mathrm{S} 1$ & & $\mathrm{X}$ & $\begin{array}{l}\text { Nuancer le manque } \\
\text { d'expertise de la } \\
\text { société civile }\end{array}$ \\
\hline $\mathrm{S} 5 \rightarrow \mathrm{S} 6$ & $\mathrm{X}$ & $\mathrm{X}$ & $\begin{array}{l}\text { Disparition du } \\
\text { terme société civile } \\
\text { au profit d'une liste } \\
\text { floue } \\
\text { d'organisations }\end{array}$ \\
\hline $\mathrm{S} 6 \rightarrow \mathrm{S} 7$ & $\mathrm{X}$ & $\mathrm{X}$ & $\begin{array}{l}\text { Disparition du } \\
\text { terme société au } \\
\text { profit du terme de } \\
\text { \& voix s plus vague, } \\
\text { réduction du rôle de } \\
\text { représentation }\end{array}$ \\
\hline $\mathrm{S} 5 \rightarrow \mathrm{S} 6$ & $\mathrm{X}$ & $\mathrm{Disparition} \mathrm{du}$ \\
& & $\mathrm{X}$ & $\begin{array}{l}\text { terme \& faire } \\
\text { campagne }{ }^{\prime}, \\
\text { réduction du champ } \\
\text { d'action }\end{array}$ \\
\hline & & & \\
\hline
\end{tabular}

Tableau 2 - Processus de sélection d'information, d'énonciation et de compréhension appliqué à l'écriture des statuts (Sandrine Roginsky, François Lambotte)

\begin{tabular}{|l|l|l|l|}
\hline Versions & $\begin{array}{l}\text { Sélection } \\
\text { d'information }\end{array}$ & $\begin{array}{l}\text { Sélection } \\
\text { d'énonciation }\end{array}$ & $\begin{array}{l}\text { Sélection de } \\
\text { compréhension }\end{array}$ \\
\hline $\begin{array}{l}\mathrm{S} 3 \rightarrow \mathrm{S} 4 \rightarrow \mathrm{S} \\
5\end{array}$ & $\mathrm{X}$ & $\begin{array}{l}\text { Disparition des } \\
\text { particuliers comme } \\
\text { membres }\end{array}$ \\
\hline $\mathrm{S} 4 \rightarrow \mathrm{S} 5$ & $\mathrm{X}$ & $\mathrm{X}$ & $\begin{array}{l}\text { Réapparition des } \\
\text { particuliers comme } \\
\text { \& amis de FW },\end{array}$ \\
\hline $\mathrm{S} 5 \rightarrow \mathrm{S} 6 \rightarrow$ & $\mathrm{X} 7$ & & $\begin{array}{l}\text { Précision du rôle } \\
\text { des particuliers }\end{array}$ \\
\hline $\mathrm{S} 7 \rightarrow \mathrm{S} 8$ & $\mathrm{X}$ & & $\begin{array}{l}\text { Disparition de ces } \\
\text { précisions } \\
\text { concernant le rôle } \\
\text { des particuliers } \\
\text { (flou) }\end{array}$ \\
\hline & & & \\
\hline
\end{tabular}

Tableau 3 - Processus de sélection d'information, d'énonciation et de compréhension appliqué à l'écriture des statuts relatifs à la place du public

(Sandrine Roginsky, François Lambotte) 
- La disparition des élus européens

Avec la société civile et les citoyens, une autre catégorie d'acteurs disparaît au fil du processus d'écriture. Il s'agit cette fois des élus européens, à l'origine de l'appel pour Finance Watch. Leur disparition est moins linéaire : au fil des documents, ils disparaissent et réapparaissent. La présence des députés est surtout visible dans le "comité de liaison » : il s'agit d'un projet d'instance qui doit se situer directement dans l'organisation, en capacité de reconnaître et structurer la participation des députés européens en son sein. Lidée d'un « comité de liaison » est lancée par les députés européens et reprise ensuite dans les versions successives des statuts, avec au départ une coloration particulière puisque les élus sont alors identifiés comme acteurs nécessaires à la valorisation du travail d'expertise de l'organisation. Ce rôle va disparaître, même si le comité va, lui, rester et survivre aux différentes versions et discussions des statuts. Pourtant, alors qu'il semble se dégager un consensus à son encontre, il n'est pas repris dans la version finale et définitive : le comité de liaison avec les députés européens ne verra donc pas le jour, sans qu'on ne puisse en comprendre la raison par la seule analyse des écrits. Cependant leur soudaine disparition traduit bien l'idée développée par Luhmann : pour avoir une chance de perdurer comme système de communication - articulant les trois cycles textes-conversations (appel, statut et dossier presse) - et permettre la création de l'organisation, il a fallu rejeter certaines idées.

\begin{tabular}{|l|l|l|l|}
\hline Versions & $\begin{array}{l}\text { Sélection } \\
\text { d'information }\end{array}$ & $\begin{array}{l}\text { Sélection } \\
\text { d'énonciation }\end{array}$ & $\begin{array}{l}\text { Sélection de } \\
\text { compréhension }\end{array}$ \\
\hline $\mathrm{S} 1 \rightarrow \mathrm{S} 2$ & $\mathrm{X}$ & & $\begin{array}{l}\text { Rôles des élus : } \\
\text { pourvoyeurs de fonds }\end{array}$ \\
\hline $\mathrm{S} 1 \rightarrow \mathrm{S} 2$ & $\mathrm{X}$ & & $\begin{array}{l}\text { Rôles des élus : comité de } \\
\text { liaison }\end{array}$ \\
\hline $\mathrm{S} 2 \rightarrow \mathrm{S} 3$ & $\mathrm{X}$ & $\begin{array}{l}\text { Rôles des élus : comité de } \\
\text { liaison nécessaire comme } \\
\text { relai de l'expertise }\end{array}$ \\
\hline $\mathrm{S} 5 \rightarrow \mathrm{S} 6$ & $\mathrm{X}$ & & $\begin{array}{l}\text { Rôles des élus : celui de } \\
\text { supporter de FW }\end{array}$ \\
\hline $\mathrm{S} 6 \rightarrow \mathrm{S} 7$ & $\mathrm{X}$ & $\begin{array}{l}\text { Rôles des élus : celui de } \\
\text { supporter de FW affiché } \\
\text { publiquement }\end{array}$ \\
\hline $\mathrm{S} 7 \rightarrow \mathrm{S} 8$ & $\mathrm{X}$ & $\mathrm{X}$ & $\begin{array}{l}\text { Mise en schéma du rôle } \\
\text { des élus comme comité de } \\
\text { liaison }\end{array}$ \\
\hline $\mathrm{S} 8 \rightarrow \mathrm{SF}$ & $\mathrm{X}$ & $\begin{array}{l}\text { Disparition des élus } \\
\text { comme comité de liaison }\end{array}$ \\
\hline
\end{tabular}

Tableau 4 - Processus de sélection d'information, d'énonciation et de compréhension appliqué à l'écriture des statuts relatifs aux rôles des élus dans l'organisation (Sandrine Roginsky, François Lambotte) 
- le rôle central de l'expertise

C'est là un trait identitaire de l'organisation qui est présent dès la conception de l'organisation jusqu'à son élaboration plus concrète. Dès le premier document de travail, elle devient le focus de l'écriture et le restera jusqu'à la fin. Ce document détaille d'ailleurs le pôle expertise de l'organisation, qu'il place donc au centre de son fonctionnement. On trouve ainsi le schéma suivant :

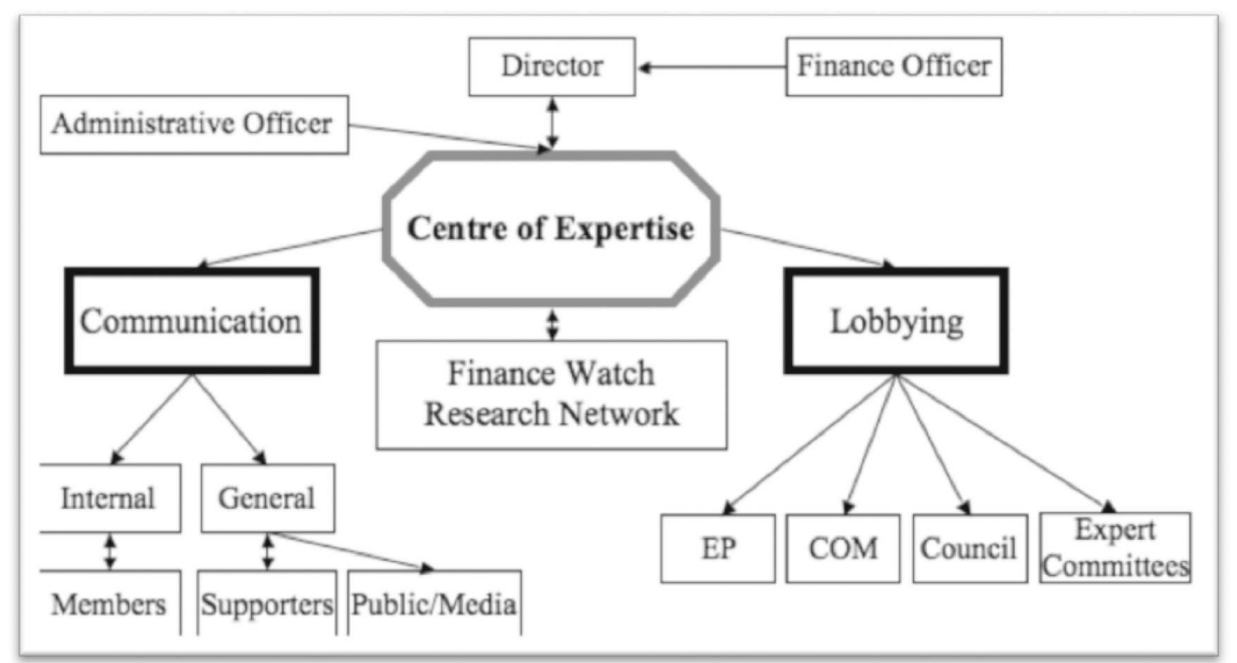

Figure 3 - Présentation de l'organisation, document S1 (Sandrine Roginsky, François Lambotte)

Dans la version suivante, une large place est donnée à la définition et à la mise en place de la démarche d'expertise. Le lobbying et la communication sont les deux autres actions de l'organisation mais sont présentées avec moins de détails, et c'est là une constante qu'on retrouve dans l'ensemble des documents étudiés. Les trois piliers initiaux se transforment finalement en deux piliers : expertise d'un côté et "advocacy » ou plaidoyer de l'autre. Le plaidoyer repose lui-même sur deux actions identifiées : le lobbying et la communication. Mais l'action de communication prend une place de plus en plus réduite dans les documents. Si les actions relatives à la production et la diffusion d'expertise sont détaillées avec précision tout au long du processus d'écriture de l'organisation, celles liées à la communication proprement dite ne font pas l'objet d'explications fournies. 


\begin{tabular}{|c|c|c|c|}
\hline Versions & $\begin{array}{l}\text { Sélection } \\
\text { d'information }\end{array}$ & $\begin{array}{l}\text { Sélection } \\
\text { d'énonciation }\end{array}$ & $\begin{array}{l}\text { Sélection de } \\
\text { compréhension }\end{array}$ \\
\hline S1 & $\mathrm{X}$ & & $\begin{array}{l}\text { FW comme expert à } \\
\text { côté des piliers } \\
\text { lobby et } \\
\text { communication }\end{array}$ \\
\hline $\mathrm{S} 1 \rightarrow \mathrm{S} 2$ & $\mathrm{X}$ & & $\begin{array}{l}\text { FW comme expert : } \\
\text { sélection et } \\
\text { descriptions des } \\
\text { profils d'experts }\end{array}$ \\
\hline $\mathrm{S} 2 \rightarrow \mathrm{S} 8$ & $\mathrm{X}$ & & $\begin{array}{l}\text { FW comme expert: } \\
\text { création de deux } \\
\text { comités d'experts }\end{array}$ \\
\hline $\mathrm{S} 8 \rightarrow \mathrm{SF}$ & $\mathrm{X}$ & & $\begin{array}{l}\text { FW comme expert: } \\
\text { disparition des deux } \\
\text { comités d'experts }\end{array}$ \\
\hline $\mathrm{S} 4 \rightarrow \mathrm{S} 9$ & $\mathrm{X}$ & $\mathrm{X}$ & $\begin{array}{l}\text { Diminution } \\
\text { progressive de la } \\
\text { mission de } \\
\text { communication. }\end{array}$ \\
\hline $\mathrm{S} 1 \rightarrow \mathrm{S} 9$ & $\mathrm{X}$ & & $\begin{array}{l}\text { Disparition du rôle } \\
\text { de FW comme } \\
\text { acteur direct de la } \\
\text { communication }\end{array}$ \\
\hline
\end{tabular}

Tableau 5 - Processus de sélection d'information, d'énonciation et de compréhension appliqué à l'écriture des statuts relatifs à la déclinaison de la notion d'expertise (Sandrine Roginsky, François Lambotte)

En résumé, l'analyse des statuts montre que si les objectifs poursuivis par l'organisation sont identiques entre la première et la dernière version des statuts, à savoir fournir de l'expertise aux membres de l'organisation et informer les individus qui soutiennent l'action de l'organisation, la manière d'y parvenir se dégage au fur à mesure du processus d'écriture des formules les plus fortes et engageantes, telles "direct actions ", "civil-society community", " wider public». Ainsi, il n'y a plus aucune trace de ces notions dans les statuts officiels qui viendront clore le processus d'élaboration. Les acteurs disparaissent des statuts. La seule dénomination d'acteurs que l'on trouve dans les différentes versions des statuts est celle de «membre » : ce qui ne donne pas beaucoup de précisions quant à la nature de ces membres. 


\section{Le dossier de presse : Lissage du discours et réapparition des acteurs}

Nous avons pu identifier deux versions du dossier de presse, le schéma suivant indiquant le processus d'écriture ( $\mathrm{P}$ pour Dossier de presse).

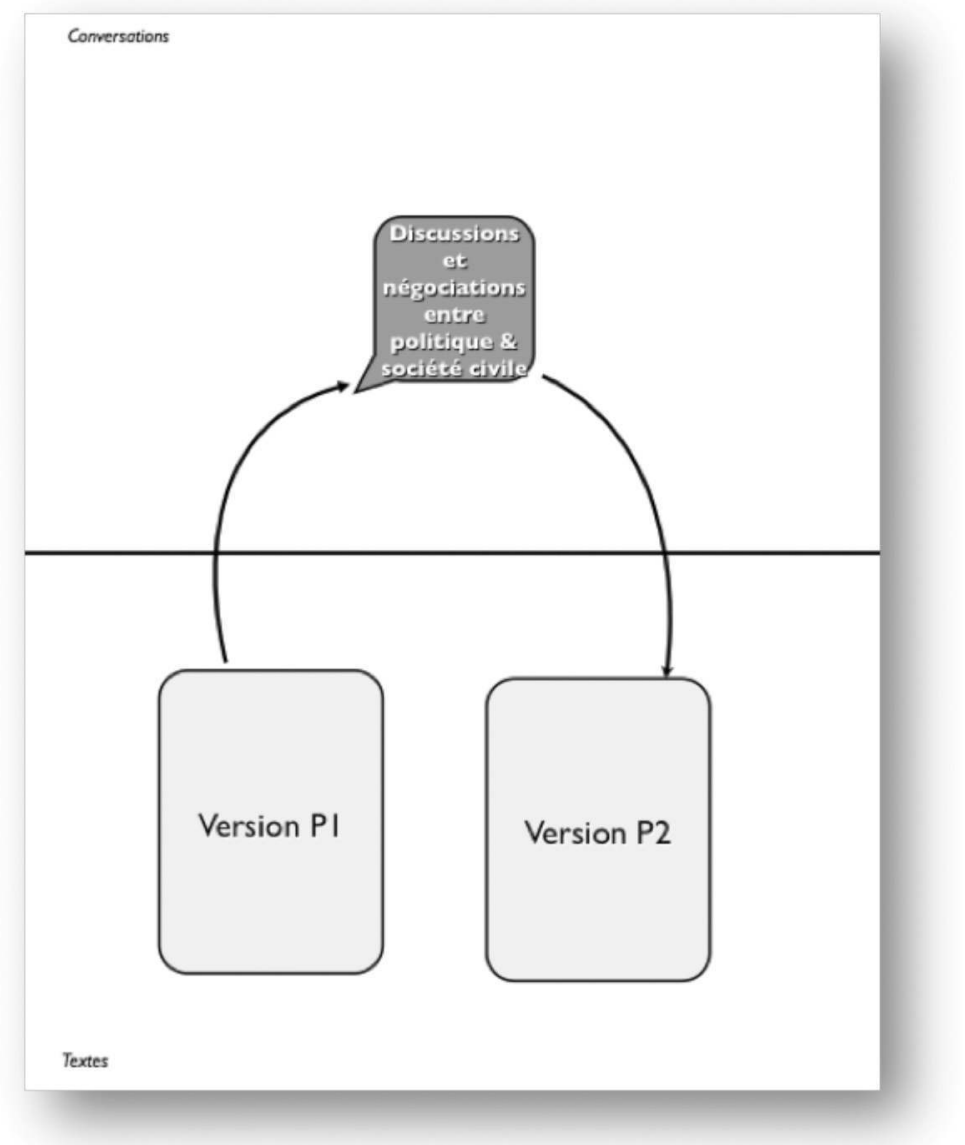

Figure 4 : Cycle texte-conversation reconstitué

pour l'écriture du dossier de presse (Sandrine Roginsky, François Lambotte)

Comme pour les statuts, on remarque une forme de lissage dans l'écriture du dossier de presse. Ce lissage se manifeste par exemple au niveau de l'argument de représentativité de l'organisation. Comme dans l'appel où l'argument du nombre pour légitimer l'initiative avait été finalement supprimé, ici aussi l'argument du nombre pour légitimer l'organisation (le nombre d'organisations de la société civile représentées par Finance Watch) est finalement enlevé.

On note une forme de dichotomie entre les statuts, qui constituent la matrice de l'organisation, et le discours public présenté dans le dossier de presse. En effet, dans ce dernier document, les missions de Finance Watch 
sont au nombre de trois : expertise, lobbying et communication. Qui plus est, elles sont présentées à égale mesure. Une autre différence notable est la place que prend ici le "public ", puisque la communication doit servir «le débat public » - autrement dit le débat général et non réservé à des spécialistes - ce qui n'est pas le cas dans les statuts. Autrement dit l'image que construit le dossier presse de l'organisation ne correspond pas complètement à ses caractéristiques internes présentées dans les statuts; elle rappelle davantage l'appel. On voit en effet ré-apparaître une forme de triptyque dans la communication, qui n'est pas présent dans les statuts mais l'est dans l'appel : la société civile, le public, les élus.

\begin{tabular}{|l|l|l|l|}
\hline Versions & $\begin{array}{l}\text { Sélection } \\
\text { d'information }\end{array}$ & $\begin{array}{l}\text { Sélection } \\
\text { d'énonciation }\end{array}$ & $\begin{array}{l}\text { Sélection de } \\
\text { compréhension }\end{array}$ \\
\hline $\mathrm{P} 1 \rightarrow \mathrm{P} 2$ & & $\mathrm{X}$ & $\begin{array}{l}\text { Lissage du texte } \\
\text { concernant } \\
\text { l'inexistence d'une } \\
\text { onG dans le secteur } \\
\text { bancaire }\end{array}$ \\
\hline $\mathrm{P} 1 \rightarrow \mathrm{P} 2$ & $\mathrm{X}$ & & $\begin{array}{l}\text { FW comme } \\
\text { représentant de la } \\
\text { société civil : } \\
\text { disparition des } \\
\text { nombres. }\end{array}$ \\
\hline $\mathrm{P} 2$ \& S9 & $\mathrm{X}$ & $\mathrm{X}$ & $\begin{array}{l}\text { Communication } \\
\text { équilibrée entre les } \\
\text { trois missions : } \\
\text { expertise, lobbying } \\
\text { et communication }\end{array}$ \\
\hline $\mathrm{P} 2$ \& S9 & $\mathrm{X}$ & $\begin{array}{l}\text { Réapparition des } \\
\text { acteurs société } \\
\text { civile, public et élu }\end{array}$ \\
\hline
\end{tabular}

Tableau 6 - Processus de sélection d'information, d'énonciation et de compréhension appliqué à l'écriture du dossier de presse

(Sandrine Roginsky, François Lambotte)

Finalement, l'analyse du jeu de sélection d'information et d'énonciation permet de faire émerger plusieurs éléments qui, par leur mise en visibilité ou leur occultation, participent à la construction de l'identité de Finance Watch. Ainsi l'analyse montre comment se façonnent progressivement l'identité, le rôle et le champ d'action de l'organisation mais également les relations entre l'organisation, la société civile et les élus. On voit en effet les sujets qui prennent de l'ampleur et ceux qui disparaissent ; ce qui permet de mettre en avant la dynamique des négociations qui ont lieu autour des documents 
dans une approche longitudinale. Les processus de modifications des textes permettent la survie de la communication et la création de l'organisation. Leur analyse permet également de montrer comment se renforce progressivement la mission d'expertise par rapport aux axes de lobbying et de communication.

\section{Discussion : la dilution des auteurs et des acteurs}

En même temps que l'énonciation de l'organisation fait disparaître les acteurs en présence, en tout cas leur dénomination, elle pratique également une dilution des auteurs. En effet, les textes sont pour leur grande majorité sans auteur et on ne sait d'ailleurs pas qui est partie prenante du processus d'écriture. L'énonciation semble ne plus appartenir à personne. Ce qui rend par exemple difficile d'évaluer la confidentialité du processus d'écriture, ou au contraire son ouverture. Il y a une forme d'occultation de l'ensemble des personnes qui ont ainsi participé à l'élaboration de l'organisation, qu'il s'agisse des organisations de la société civile ou des élus du Parlement européen.

Cette démarche d'occultation, révélée dans l'analyse par l'apparition puis la disparition de certaines thématiques, nous semble participer à la construction même de l'organisation, elle se révèle nécessaire pour établir une forme de consensus entre des acteurs aux objectifs divergents, voire concurrents, et donner ainsi de la légitimité à l'organisation. Elle permet également de gagner en marge de manœuvre puisqu'elle évite ainsi des négociations difficiles voire impossibles à résoudre. Elle participe par ailleurs au lissage du discours. À ce titre, et bien qu'il ne s'agisse pas ici d'un discours d'institution à proprement parler, son énonciation suit malgré tout un processus similaire, tel que décrit par Ollivier-Yaniv et Oger (2006) : il s'agit en effet ici aussi d'une " énonciation collective négociée ", qui s'accompagne " d'un effort de réduction de l'hétérogène à l'homogène ". À ce titre, le fait que les acteurs qui débutent l'écriture de l'organisation soient originaires d'institutions n'est peut-être pas anodin. Un tel mouvement conduit à une forme d'injonction contradictoire, celle de "neutraliser » au maximum l'écriture donc gommer les nuances et les divergences tout en promouvant la transparence dans le fonctionnement et l'objectif de l'organisation. En effet Finance Watch a fait de la transparence un de ces trois principes de fonctionnement, à côté de l'indépendance et de la bonne gouvernance. Elle en a également fait un objectif puisqu'elle plaide pour un système financier équitable, durable et transparent. La notion de transparence est donc centrale pour l'ONG. Cependant, on peut se demander si l'objectif de transparence de l'organisation ne s'accompagne pas d'un impératif - l'expertise pointue dans le domaine financier - qui fonctionne concrètement comme une limitation drastique de l'expression claire et transparente. L'expertise se veut accessible et transparente mais ne peut véritablement l'être : on voit ainsi tout au long du processus d'écriture disparaître de l'organisation les «non spécialistes » de la finance, autrement dit le grand public et les élus. La transparence serait ainsi d'abord une question 
de "face ", pour reprendre le terme de Goffman, la mise en scène d'une organisation qu'il faut alors voir comme une " équipe de représentation ». Ainsi la construction de l'image publique ("la face ») de l'organisation ne correspond pas spécifiquement à ses caractéristiques internes : le dossier de presse met en avant des éléments qui sont gommés dans les statuts. Ce qui fait cohérence, malgré tout, et qu'on retrouve à la fois dans le discours public (l'appel et le dossier de presse) et le discours privé (les versions successives des statuts) est la place centrale de l'expertise. Elle participe pleinement au processus de lissage dans l'écriture de l'organisation. En effet, l'analyse de l'ensemble des documents écrits montre que l'écriture procède par petites touches à une forme de consensualisation de l'organisation.

\section{Conclusion}

Le monde de la finance est souvent caractérisé par son opacité et sa complexité. C'est dans ce contexte que l'ONG Finance Watch voit le jour, à l'initiative de députés européens. Finance Watch est aujourd'hui une association dont l'objectif est de faire prendre en compte la dimension d'intérêt général de l'industrie financière. Sa mission est de renforcer la voix de la société civile et de la faire peser dans les réformes de la règlementation financière, essentiellement européenne mais également déclinée au niveau national dans l'Union européenne. La dynamique de légitimation de l'organisation passe notamment par une communication centrée sur la nécessité d'une expertise accessible, indépendante et transparente. L'analyse du processus d'écriture de l'organisation permet ainsi de mettre en avant la construction identitaire de l'organisation centrée sur l'expertise. Pour autant, nous remarquons que l'expertise prend finalement le pas sur les autres éléments : nous constatons ainsi une disparition des auteurs et des acteurs. Cette disparition permet à l'organisation de prendre ses distances avec les politiques qui l'ont portée, mais également, de manière plus étonnante, avec le " grand public ». La société civile, elle aussi, semble disparaitre des traces écrites en laissant finalement planer un certain flou sur les acteurs qui constituent l'organisation. Ces éléments participent à produire une forme d'opacité qui contraste avec la rhétorique de transparence de l'organisation. En reconstituant chaque étape du développement de l'organisation, il est ainsi possible de faire ressortir ce qui est communicable et ce qui n'est pas. L'analyse des traces écrites de l'organisation, en particulier des documents participant à l'élaboration des statuts, permet en effet de voir comment ceux-ci ont participé à façonner Finance Watch et d'éclairer la manière dont l'organisation est labellisée, en contrastant l'écriture du discours à visée interne et l'écriture du discours à visée externe. Le fait même que discours interne et discours externe ne coïncident pas complètement nous semble être l'illustration là aussi des limites de la rhétorique de la transparence. L'écriture est par conséquent considérée dans cette recherche comme une activité déterminante à la fois stratégique 


\section{$\mathrm{C} \& O \mathrm{n}^{\circ} 45$}

et invisible (Delcambre, 1997). À ce titre, l'analyse des traces écrites nous permet ici d'opérationnaliser les concepts développés par Luhmann (1992) en s'intéressant aux communications écrites à la genèse de l'organisation qui nous permettent ainsi de saisir l'action de constitution de l'organisation. Comme le rappelle Huët (2012), tout texte écrit est "le résultat d'un processus dans lequel se jouent des activités complexes de coopération entre les acteurs par la discussion, des négociations de place pour les mettre en forme, etc. ». Les énonciateurs se trouvent finalement confrontés au paradoxe d'une transparence certes promue et valorisée mais qui n'en demeure pas moins enserrée dans un ensemble de contraintes et de normes (Oger, 2003) : la négociation entre différents acteurs, associatifs et politiques qui plus est, aux objectifs parfois concurrents ; l'obligation de démontrer sa légitimité (l'expertise) qui restreint en même temps l'espace de mobilisation de l'organisation, les modalités de financement, etc. On voit donc apparaittre une forme de tension entre une transparence prescrite et la clôture constitutive d'une organisation. Notre analyse témoigne des limites de la rhétorique de transparence. En effet, l'opacité n'est pas (nécessairement) le mensonge mais seulement une forme communicationnelle particulière.

\section{BIBLIOGRAPHIE}

ASHCRAFT K. L., KUHN T. R., COOREN F., « Constitutional Amendments: "Materializing" Organizational Communication ", The Academy of Management Annals, 3(1), 2009, p. 1-64.

DELCAMBRE P., «Écritures professionnelles et pouvoirs : Analyse d'un dispositif d'écriture ", Études de communication. Langages, information, médiations (11), 1990(a), p. 13-53.

DELCAMBRE P., «Une analyse d'écriture revisitée : ou, quand l'analyse d'écriture fait intervention, que dire de ses effets? ", Études de communication. Langages, information, médiations (11), 1990(b), p. 131-150.

DELCAMBRE P., Écriture et communication de travail : pratiques d'écriture des éducateurs spécialisés. Villeneuve d'Ascq : Presses universitaires du septentrion, 1997.

GOFFMAN E., La mise en scène de la vie quotidienne. La représentation de soi. Paris : Les Éditions de Minuit, 1959.

HUET R., La fabrique de l'éthique. Les nouvelles promesses des entreprises. Paris : CNRS Alpha, 2012.

HUET R., LONEUX C., " Les nouvelles questions économiques et sociales à l'épreuve de l'analyse communicationnelle ", Congrès de la SFSIC, 2012, http://www. sfsic.org/congres_2008/spip.php?article67

LUHMANN N., "What is communication ? », in MULLER, H. L., \& CRAIG, R.T. (Eds.), Theorizing communication readings across traditions, Sage Publications, 2007, p. 301-307. 
OGER C., « Communication et contrôle de la parole : de la clôture à la mise en scène de l'institution militaire ", Quaderni. 52. Secret et pouvoir : les faux-semblants de la transparence, 2003, p. 77-92.

OGER C., OLLIVIER-YANIV C., « Conjurer le désordre discursif. Les procédés de « lissage " dans la fabrication du discours institutionnel ", Mots. Les langages du politique. 81, 2006, p. 63-77.

PENE S., "Les écrits et les acteurs. Circulation des discours et empreinte des objets », Études de communication. Langages, information, médiations (16), 1995, p. 57-75.

SEIDL D., «The basic concepts of Luhmann's theory of social systems ». In D. SEIDL \& K. H. BECKER (Eds.), Niklas Luhmann and organization studies (p. 21-53). Oslo, Norway: Copenhagen Business School Press, 2005(a).

SEIDL D., "Organization and interaction », in D. SEIDL \& K. H. BECKER (Eds.), Niklas Lubmann and organization studies (p. 145-170). Oslo, Norway: Copenhagen Business School Press, 2005(b)

SCHOENEBORN D., "Organization as Communication A Luhmannian Perspective ", Management Communication Quarterly, 25(4), 2011, p. 663-689.

TAYLOR J R., COOREN F., GIROUX N., ROBICHAUD D., "The Communicational Basis of Organization: Between the Conversation and the Text ", Communication Theory, 6(1), 1996, p. 1-39.

Résumé : Finance Watch est une association née en juin 2011 à Bruxelles pour représenter la société civile face au lobby de l'industrie financière. À partir de l'analyse du cycle d'écriture des documents fondateurs de l'organisation, il est possible de raconter l'histoire de la création, c'est-à-dire de montrer comment se façonnent progressivement l'identité, le rôle et le champ d'action de l'organisation mais également ses relations avec la société civile, les élus et les particuliers. Une telle démarche permet de mettre en avant les paradoxes et les contradictions entre la rhétorique de la transparence que porte l'organisation et l'opacité que nécessite sa construction. Celle-ci s'inscrit dans l'approche $\mathrm{CCO}$ considérant la communication constitutive de l'organisation et s'appuie sur la conception systémique de la communication de Luhmann.

Mots-clés : Écriture, énonciation, discours, texte-conversation, transparence, société civile, expertise

Abstract: Finance Watch is a non-profit association set up in June 2011 to act as a public interest counterweight to the powerful financial lobby. The analysis of the founding documents' writing process makes it possible to tell the story of the creation of the organisation, especially the construction of its identity, its role and the field of its action as well as its relationships with civil society, elected members of the European Parliament and individuals. Such an approach highlights the paradoxes and contradictions between the rhetoric of transparency of the organization and the necessary opacity needed to build up the organization. It fits into the CCO theoretical framework (communication constitutes organization) and builds on Lubmann's systems theory of communication.

Keywords: Discourse, text-conversation, transparency, civil society, expertise 


\title{
Annexe 1 : Exemple de comparaison entre deux documents
}

\author{
Version A1
}

En tant qu'élus européens et américains en charge de la régulation financière nous appelons done de nos vœux la création d'une (ou plusieurs) organisation non gouvernementale capable(s) de développer une contre expertise sur les activités menées sur les marchés financiers par les principaux opérateurs (banques, compagnies d'assurances, hedge funds, etc.ara). Une (ou des) organisation qui devra également être capable de faire connaître cette contre-expertise de manière pédagogique au moyen d'outils de sensibilisation du grand public et des medias. En tant qu'élus issus de plusieurs familles politiques et de plusieurs pays nous sommes prêts à contribuer à la constitution d'une telle ONG sans bien entendu chercher à la contrôler ce qui serait contraire à notre vision de la démocratie.

Pour être à la fois représentative, efficace et indépendante, cette ONG (ou ces ONG) a besoin d'avoir un socle important d'adhésions dans la société civile. Nous invitons donc tout ceux qui pensent que cette initiative est nécessaire à envoyer une promesse d'adhésions en se connectant sur XXXXXX. Nous invitons également les élus qui ne sont pas satisfaits de la situation actuelle à signer cet appel sur le même site internet.

Si cet appel suscite plus de 100000 promesses d'adhésion en Europe et aux Etats-Unis, nous considérerons que le socle de légitimité est atteint et nous travaillerons alors avec l'ensemble des organisations de la société civile qui le souhaitent à la création effective de ce chaînon manquant de la démocratie.

\section{Version A2 (version définitive)}

En tant qu'élus européens en charge de la réglementation financière et bancaire nous appelons donc la société civile (QNGs, syndicats, universitaires, think-tanks...) à s'organiser pour créer une (ou plusieurs) organisation non gouvernementale capable(s) de développer une contre expertise sur les activités menées sur les marchés financiers par les principaux opérateurs (banques, compagnies d'assurances, hedge funds, etc.ese) et de faire connaitre de manière efficace cette analyse aux medias. En tant qu'élus issus de plusieurs familles politiques nous pouvons diverger sur les mesures à prendre. Mais nous convergeons pour alerter l'opinion sur ce risque pour la qualité de la démocratie. En tant que parlementaires européens, nous invitons les élus des parlements nationaux à rejoindre notre appel en se rendant sur le site internet dédié www.finance-watch.org. 